\title{
Comparative Genomic Analysis and Phenotypic Characterization of Bronchoscope-Associated Klebsiella aerogenes
}

\author{
FANG HUANG ${ }^{1 *}$, SHUANG LI' ${ }^{2,3 *}$, LAN LOU $^{1}, J_{U N J U N ~ M O}{ }^{1 *}$ and HAO XU ${ }^{3 *}$ \\ ${ }^{1}$ Disinfection and Sterilization Center, The First Affiliated Hospital, College of Medicine, \\ Zhejiang University, Hangzhou, China \\ ${ }^{2}$ Department of Laboratory Medicine, The First Affiliated Hospital of Zhengzhou University, \\ Zhengzhou, China \\ ${ }^{3}$ Collaborative Innovation Center for Diagnosis and Treatment of Infectious Diseases, \\ State Key Laboratory for Diagnosis and Treatment of Infectious Diseases, The First Affiliated Hospital, \\ College of Medicine, Zhejiang University, Hangzhou, China
}

Submitted 6 June 2021, revised 21 July 2021, accepted 4 August 2021

\begin{abstract}
Bronchoscopes have been linked to outbreaks of nosocomial infections. The phenotypic and genomic profiles of bronchoscope-associated Klebsiella aerogenes isolates are largely unknown. In this work, a total of 358 isolates and 13 isolates were recovered from samples after clinical procedures and samples after decontamination procedures, respectively, over the five months. Antimicrobial susceptibility testing found seven $K$. aerogenes isolates exhibiting a low-level resistance to antimicrobial agents. Among seven K. aerogenes isolates, we found five sequence types (STs) clustered into three main clades. Collectively, this study described for the first time the phenotypic and genomic characteristics of bronchoscope-associated K. aerogenes.
\end{abstract}

Ke y w o r d s: Klebsiella aerogenes, bronchoscope-associated, phenotypic, genomics characteristics

Flexible bronchoscopes are medical devices widely used for diagnostic and therapeutic procedures. Usually, they are heat-labile and complex, which leads to difficulty in cleaning procedures (DiazGranados et al. 2009). Patient-ready reusable, flexible bronchoscopes can be contaminated and damaged and pose a severe threat to patient safety (Zamani 2004). It is well documented in the literature that some nosocomial outbreaks have been linked to contaminated or inadequately disinfected bronchoscopes (Srinivasan et al. 2003). Moreover, recent studies determined that microbial transmission occurs even when proper cleaning and disinfection protocols are followed with standard guidelines (Galdys et al. 2019). Recently, endoscopes have been suggested as point sources of nosocomial Enterobacteriaceae infections (Gastmeier and
Vonberg, 2014). Previous investigations have shown a significant reduction of microbial contaminants with cleaning alone, and recommendations require cleaning to be performed promptly following use (Galdys et al. 2019). In China, the national standard "Regulation for Cleaning and Disinfection Technique of Flexible Endoscope (WS507-2016)" was used for disinfection of endoscopes, including the selection of chemicals to ensure quality control throughout the clinical management (Gu et al. 2020). However, the microbiology data on cleaning and disinfection of endoscopes is unclear. Moreover, bronchoscope-associated microbial profiles and phenotypic characteristics are largely unknown.

$K$. aerogenes is a Gram-negative, rod-shaped, anaerobic bacterium, a commensal microorganism living in the mouth and gut. However, the bacterium is now

\footnotetext{
\# Fang Huang and Shuang Li contributed equally to this study.

* Corresponding authors: H. Xu, Collaborative Innovation Center for Diagnosis and Treatment of Infectious Diseases, State Key Laboratory for Diagnosis and Treatment of Infectious Diseases, The First Affiliated Hospital, College of Medicine, Zhejiang University, Hangzhou, China; e-mail: xuhao0523@zju.edu.cn

J. Mo, Disinfection and Sterilization Center, The First Affiliated Hospital, College of Medicine, Zhejiang University, Hangzhou, China; e-mail: mojunjunshou@163.com

(c) 2021 Fang Huang et al.

This work is licensed under the Creative Commons Attribution-NonCommercial-NoDerivatives 4.0 License (https://creativecommons. org/licenses/by-nc-nd/4.0/).
} 
resistant to many antimicrobial agents, including one of the latest antibiotics-carbapenems, which represents a serious challenge to public health (Zheng et al. 2020). It is an important opportunistic and multi-resistant bacterial pathogen for patients during the past decades in clinical settings (Malek et al. 2019). It is widely associated with bloodstream, skin and soft tissue, respiratory, and urinary tract infections (Shen et al. 2019). Recently, the emergence of carbapenem-resistant K. aerogenes and colistin-resistant $K$. aerogenes isolates in China is of concern (Liao et al. 2020). A previous study revealed that the prevalence of carbapenem-resistant K. aerogenes isolates in a Chinese teaching hospital was caused by clonal dissemination (Qin et al. 2014). However, the prevalence, epidemiology, resistance mechanism, and genetic background of K. aerogenes in China remain largely unknown due to the limited number of investigations performed to date in this field (Miao et al. 2019; Ma et al. 2020).

The aims of the current study were to evaluate the microbial profiles of endoscopes pre- and post-disinfection in the Disinfection and Sterilization Center, and investigate the phenotypic characteristics and genomic complexity of $K$. aerogenes strains isolated from bronchoscope samples.

From January 2019 to May 2019, the study was conducted in the Disinfection and Sterilization Center of the First Affiliated Hospital, School of Medicine, Zhejiang University, where both gastrointestinal and respiratory endoscopes are reprocessed. During the study period, procedures in our institution were performed using bronchoscopes (model BF260) (Olympus, Japan). The cleaning of bronchoscopes was carried out with an enzymatic detergent solution, endozyme. Manual disinfection was performed by soaking the device into $2 \%$ glutaraldehyde for 30 minutes.

Samples were collected under aseptic conditions from bronchoscopes following clinical procedures and after usual decontamination procedures by flushing thoroughly with $10 \mathrm{ml}$ of sterilized phosphate-buffered saline (PBS) and shaking for 30 seconds, as described previously (Jørgensen et al. 2016). Collected samples were put in cool boxes with ice packs $\left(4-8^{\circ} \mathrm{C}\right)$ upon collection and transported within 4 hours to the laboratory.

All samples $(100 \mu l)$ were plated on Mueller-Hinton agar plates (Oxoid, UK) using the sterile swab. The agar plates were incubated for $18-24$ hours at $37^{\circ} \mathrm{C}$. A single colony was selected from each species per sample. All of the positive cultures were selected for identification. Bacterial identification was conducted by matrixassisted laser desorption/ionization-time of flight mass spectrometry (MALDI-TOF-MS) (Bruker, Leipzig, Germany) and further checked by PCR and sequencing.

The minimum inhibitory concentrations (MICs) of seven K.aerogenes isolates were determined using the agar dilution method according to the Clinical and Laboratory Standards Institute (CLSI) standards (Zheng etal. 2015). Nineteen antimicrobials were tested as described previously (Zheng et al. 2015). Antimicrobial susceptibility testing for colistin and tigecycline was performed using the microbroth dilution method described by the European Committee on Antimicrobial Susceptibility Testing (EUCAST). The MIC results were interpreted using the CLSI standards (Third Edition: M45).

WGS was performed on all $K$. aerogenes strains identified in this work. The extracted genomic DNA was evaluated by agarose gel electrophoresis. The concentration and purity of genomic DNA were determined using NanoDrop 2000 (Thermo Scientific, Waltham, USA) and Qubit $^{\oplus}$ version 2.0 fluorometer (Thermo Scientific), respectively. The sequencing library was prepared with the Illumina Nextera XT kit (Illumina, San Diego, USA). A-tailed fragments were ligated with paired-end adaptors and PCR-amplified with a 500-bp insert. WGS was performed using an Illumina NovaSeq 6000 platform (Novogene Co., China). PCR adapter reads and low-quality reads from the paired-end and mate-pair library were filtered using an in-house pipeline. Paired reads were then assembled into many scaffolds using Velvet version 1.2.10 (Zerbino and Birney, 2008). Multilocus sequence typing (MLST) analysis was performed as described previously (Cerqueira et al. 2017). Antibiotics Resistance Genes (ARGs) were identified using the ResFinder 4.1 database (https://cge.cbs. dtu.dk/services/ResFinder) (Zankari et al. 2012).

To further characterize the evolutionary relationship among $K$.aerogenes isolates, we created a core genome-based phylogenetic tree using seven K. aerogenes genomes sequenced in this study and 51 randomly selected publicly available K. aerogenes genomes (Table SI). The isolate collection included strains from humans $(n=44)$, the environment $(n=9)$, and other sources $(n=5)$ widely distributed over time and geographical locations. All collection genomes were annotated using Prokka (https://github.com/tseemann/ prokka) and the RAST tool (https://rast.nmpdr.org). The core genes in $K$. aerogenes genomes were identified using Prokka, and maximum likelihood-based phylogenetic reconstruction was performed with Roary (https://github.com/yikedou/Roary). One hundred bootstrap replicates were evaluated to determine branch support. A maximum-likelihood phylogenetic tree based on the core single nucleotide polymorphism alignments was generated using FastTree (Price et al. 2009). Phylogenetic tree visualizations were produced using the Interactive Tree of Life (https://itol.embl.de).

Over the five months, 250 bronchoscopes were sampled, and 500 samples were collected in a single cycle, including 250 samples after clinical procedures and 250 samples after usual decontamination procedures. 


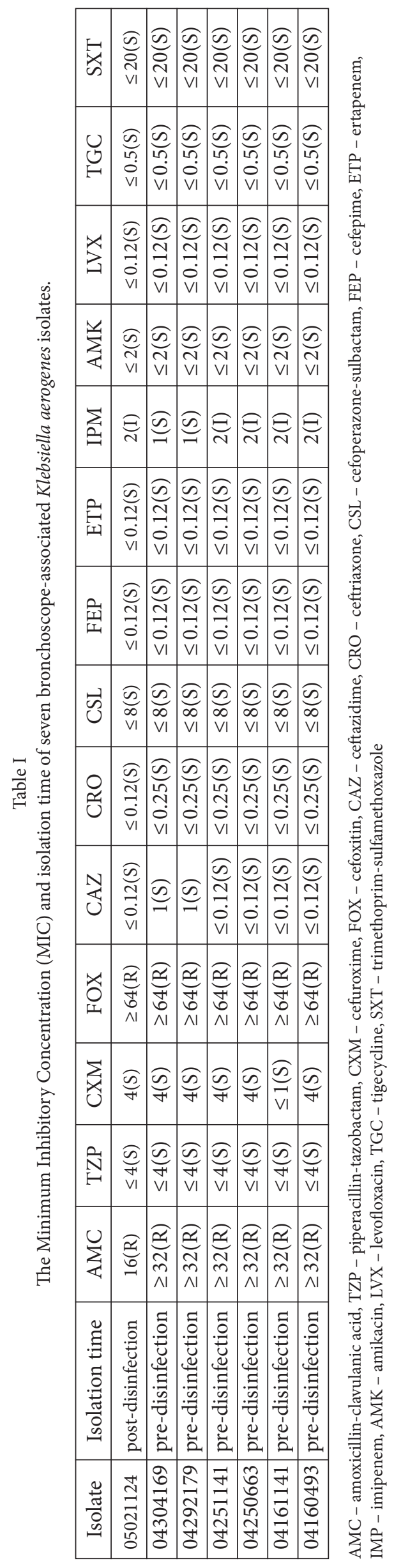

All bronchoscope samples were tested for bacteria. A total of 358 isolates and 13 isolates were recovered from samples after clinical procedures and samples after decontamination procedures, respectively (Table SII and Table SIII). Of note, most of the detected microorganisms were Gram-positive bacteria, such as Staphylococcus epidermidis $(\mathrm{n}=69)$, Streptococcus salivarius $(\mathrm{n}=42)$, and Streptococcus oralis $(\mathrm{n}=23)$. Among the Gram-negative bacilli isolates, most of them belong to the Enterobacterales group (Table SII and Table SIII). Moreover, K.aerogenes was found in both groups, includes six isolates before the cleaning procedure and one after disinfection. This prompted us to investigate further the phenotypic and genomic characteristics of seven $K$. aerogenes identified in this work.

Antimicrobial susceptibility of seven K. aerogenes isolates is detailed in Table I. The full resistance (100\% isolates) was observed for amoxicillin-clavulanic acid and cefoxitin (100\%). All isolates were susceptible to piperacillin-tazobactam, cefuroxime, ceftazidime, ceftriaxone, cefoperazone-sulbactam, cefepime, ertapenem, amikacin, levofloxacin, tigecycline, and trimethoprimsulfamethoxazole. Interestingly, five isolates were intermediate to imipenem. Among seven K. aerogenes isolates, we found five sequence types (STs), which were ST135 $(\mathrm{n}=2)$ and ST1358 $(\mathrm{n}=2)$, followed by ST1357 $(n=1), \operatorname{ST} 1359(n=1)$, and ST1363 $(n=1)$.

The Roary matrix-based gene sequence analysis generated a large pan-genome of 18,105 gene clusters of 58 full genomes. The whole-genome phylogeny (Fig. 1) revealed a population structure that was generally concordant with MLST (data not shown). Genetic diversity was observed in our bacterial collection, which clustered into three main clades.

We identified a total of 43 antimicrobial resistance genes in the K. aerogenes core genomes (Fig. 2). The resistome of $K$. aerogenes comprises a high number of antibiotic efflux pumps as well as narrow and extendedspectrum $\beta$-lactamases. As expected, human isolates encoded more antimicrobial resistance genes than environmental isolates. Of note, three isolates from this work possessed only one resistance gene, fos $A$, which is consistent with their phenotypic characteristics.

This study assessed the phenotypic characteristics and genomic complexity of $K$. aerogenes strains isolated from bronchoscope samples. It is worthy to note that 13 strains were isolated after cleaning procedures. It might indicate their low-level contamination with environmental and skin bacteria since bronchoscope samples were collected after the clinical procedures without disinfection or cleaning processes.

It is well known that outbreaks and pseudo-outbreaks may be associated with bronchoscopes (Guy et al. 2016). These nosocomial infections are commonly associated with Mycobacterium spp. and Enterobacteriaceae 
isolates (Kirschke et al. 2003). In this work, we recovered seven $K$. aerogenes isolates from bronchoscope samples. $K$. aerogenes is associated with nosocomial infections and display multidrug resistance (Shen et al. 2019). The most prevalent STs were ST93 and ST4 (PassarelliAraujo et al. 2019a). However, we did not detect any multidrug-resistant $K$. aerogenes in this work. Furthermore, STs of $K$. aerogenes found in this work have not been described in the literature. The 2 ST1358 strains identified in this study were aggregated in 1 clade with 1 ST1364 human isolate from Spain, which suggested that ST1358 and ST1364 might originate from the same ancestor. The results also indicate that 11 ST93 isolates and 9 ST56 were clustered into one separate sub-cluster, respectively, which exhibited a slight difference in the core genome sequence. Recent studies found that ST93 was the most prevalent clone in the global $K$. aerogenes genome database, indicating that ST93 might be the dominant global clone sequence in clinical settings (Malek et al. 2019; Passarelli-Araujo et al. 2019b). Furthermore, the emergence of fecal carriage and human infection of s $K$. aerogenes isolates resistant to multiple antibiotics, especially resistant to carbapenems, is considered a substantial threat to public health (Liu et al. 2019; Tian et al. 2020).

Although phenotypic and genomic evidence from the current study revealed that isolates recovered in this work are not multi-resistant. Active surveillance of bronchoscope-associated $K$. aerogenes isolates would improve our understanding of the population structure of this species. Of note, all isolates recovered from this study have a close relation to environmental or human isolates. A recent study investigated the population structure, virulence, and antimicrobial resistance in K. aerogenes (Passarelli-Araujo et al. 2019a). Their findings showed that $K$. aerogenes has an open pangenome and a large effective population size, which is in line with our results.

This study described the phenotypic and genomic characteristics of bronchoscope-associated K.aerogenes, although the relatively small number of not multi-resistant strains identified limits this finding. The detection of seven isolates of $K$. aerogenes in the surveyed Disinfection and Sterilization Center further indicates that this opportunistic pathogen may be a source of nosocomial infections without proper disinfection protocols. These results may lead to a better understanding of the genetic background and population structure of $K$. aerogenes in clinical settings.

\section{Availability of data and materials}

All genome assemblies of K.aerogenes isolates were deposited in GenBank and are registered under BioProject accession no. PRJNA633774. The datasets generated during and/or analyzed during the current study are available in the NCBI repository,
https://www.ncbi.nlm.nih.gov/bioproject/?term=PRJNA633774. Accesion Number: JABKDA000000000-JABKDC000000000, JABKCW000000000- JABKCZ000000000.

\section{Acknowledgments}

The authors would like to thank the participants, coordinators, and administrators for their support during the study.

\section{Author contributions}

All authors made a significant contribution to the work reported, whether that is in the conception, study design, execution, acquisition of data, analysis and interpretation, or in all these areas; took part in drafting, revising or critically reviewing the article; gave final approval of the version to be published; have agreed on the journal to which the article has been submitted; and agree to be accountable for all aspects of the work.

\section{Funding}

This study was supported by funding from the National Key Research and Development Program of China (No. 2016YFD0501105); the National Natural Science Foundation of China (No. 81741098); the Mega-projects of Science Research of China (2018ZX10733402-004); and the Medical Health Science and Technology Project of Zhejiang Provincial Health Commission (No.2018ZH010). Scientific Research Fund of Zhejiang Provincial Education Department (Y202043398)

\section{Conflict of interest}

The authors do not report any financial or personal connections with other persons or organizations, which might negatively affect the contents of this publication and/or claim authorship rights to this publication.

\section{Literature}

Cerqueira GC, Earl AM, Ernst CM, Grad YH, Dekker JP, Feldgarden M, Chapman SB, Reis-Cunha JL, Shea TP, Young S, et al. Multi-institute analysis of carbapenem resistance reveals remarkable diversity, unexplained mechanisms, and limited clonal outbreaks. Proc Natl Acad Sci USA. 2017 Jan 31;114(5):1135-1140. https://doi.org/10.1073/pnas.1616248114

DiazGranados CA, Jones MY, Kongphet-Tran T, White N, Shapiro M, Wang YF, Ray SM, Blumberg HM. Outbreak of Pseudomonas aeruginosa infection associated with contamination of a flexible bronchoscope. Infect Control Hosp Epidemiol. 2009 Jun; 30(6):550-555. https://doi.org/10.1086/597235

Galdys AL, Marsh JW, Delgado E, Pasculle AW, Pacey M, Ayres AM, Metzger A, Harrison LH, Muto CA. Bronchoscopeassociated clusters of multidrug-resistant Pseudomonas aeruginosa and carbapenem-resistant Klebsiella pneumoniae. Infect Control Hosp Epidemiol. 2019 Jan;40(1):40-46.

https://doi.org/10.1017/ice.2018.263

Gastmeier P, Vonberg RP. Klebsiella spp. in endoscopy-associated infections: we may only be seeing the tip of the iceberg. Infection. 2014 Feb;42(1):15-21. https://doi.org/10.1007/s15010-013-0544-6 Gu Q, Wang H, Fang Y, Lu Y, Shen Z, Wang Y, Wu X, Cen L, Chen Y. Analysis of an improved workflow of endoscope reprocessing for bedside endoscopic diagnosis and treatment on COVID-19 patients. J Zhejiang Univ Sci B. 2020 May;21(5):416-422. https://doi.org/10.1631/jzus.B2000109

Guy M, Vanhems P, Dananché C, Perraud M, Regard A, Hulin M, Dauwalder O, Bertrand X, Crozon-Clauzel J, Floccard B, et al. Outbreak of pulmonary Pseudomonas aeruginosa and Stenotrophomonas maltophilia infections related to contaminated 


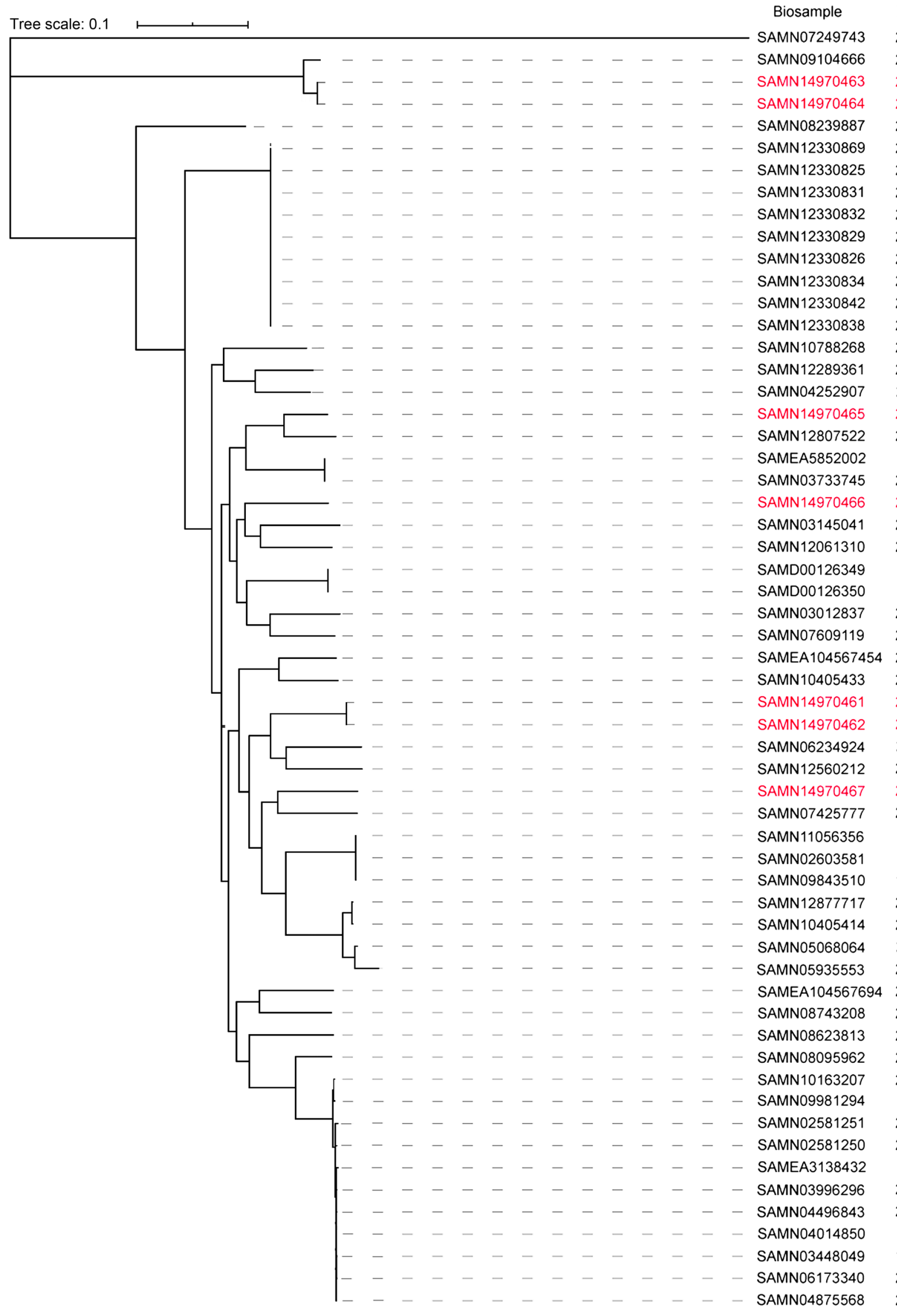

\begin{tabular}{|c|c|c|c|c|}
\hline Date & STs & Host Country & Source & source \\
\hline 2017 & 202 & & & Homo sapiens \\
\hline 2013 & 1364 & & & food \\
\hline 2019 & 1358 & & & \\
\hline 2019 & 1358 & & & environmental \\
\hline 2017 & 1366 & & & cow \\
\hline 2016 & 56 & & & plant \\
\hline 2016 & 56 & & & bronchoscope \\
\hline 2016 & 56 & & & unknown \\
\hline 2016 & 56 & & & \\
\hline 2016 & 56 & & & \\
\hline 2016 & 56 & & & location \\
\hline 2017 & 56 & & & Spain \\
\hline 2017 & 56 & & & China \\
\hline 2017 & 56 & & & Poland \\
\hline 2010 & 81 & & & Australia \\
\hline 2008 & 9 & & & Australia \\
\hline 2011 & 189 & & & Tunisia \\
\hline 2019 & 1357 & & & United Kingdom \\
\hline 2019 & 192 & & & USA \\
\hline NA & 133 & & & Malaysia \\
\hline 2010 & 133 & & & South Korea \\
\hline 2019 & 1363 & & & South Korea \\
\hline 2013 & 1370 & & & Singapore \\
\hline 2017 & 2 & & & Lebanon \\
\hline NA & 116 & & & France \\
\hline NA & 116 & & & Brazil \\
\hline 2012 & 186 & & & Argentina \\
\hline 2016 & 1362 & & & Germany \\
\hline 2018 & New & & & \\
\hline 2017 & 1360 & & & $\square$ unknown \\
\hline 2019 & 135 & & & \\
\hline 2019 & 135 & & & source \\
\hline 2011 & 1367 & & & surface \\
\hline 2015 & 1356 & & & soil \\
\hline 2019 & 1359 & & & urine \\
\hline 2016 & 1355 & & & blood \\
\hline NA & 134 & & & blood \\
\hline NA & 134 & & & sputum \\
\hline 1979 & New & & & rumen content \\
\hline 2008 & 4 & & & clinical sample \\
\hline 2015 & 4 & & & unknown \\
\hline 2011 & 2 & & & rectal swab \\
\hline 2016 & 1368 & & & Cumangut \\
\hline 2018 & 188 & & & human gut \\
\hline 2017 & 1365 & & & Perirectal \\
\hline 2015 & New & & & bitter gourd \\
\hline 2009 & 1361 & & & excreted bodily substance \\
\hline 2016 & 93 & & & faecal \\
\hline NA & 93 & & & Endotracheal aspirate \\
\hline 2013 & 93 & & & \\
\hline 2013 & 93 & & & stool \\
\hline NA & 93 & & & Wound - abdominal \\
\hline 2014 & 93 & & & catheter \\
\hline 2007 & 93 & & & $\square$ skin swab \\
\hline NA & 93 & & & $\square$ urine clean catch \\
\hline 1997 & 93 & & & - unimesiear caici \\
\hline 2015 & 93 & & & Chives \\
\hline 2014 & 93 & & & ascites \\
\hline
\end{tabular}

Fig. 1. Maximum-likelihood phylogeny of 58 representative global Klebsiella aerogenes isolates. The trees were constructed using Roary software.

The tips of branches are colored according to hosts, countries, and sources. Red words indicate the strains in this study. 


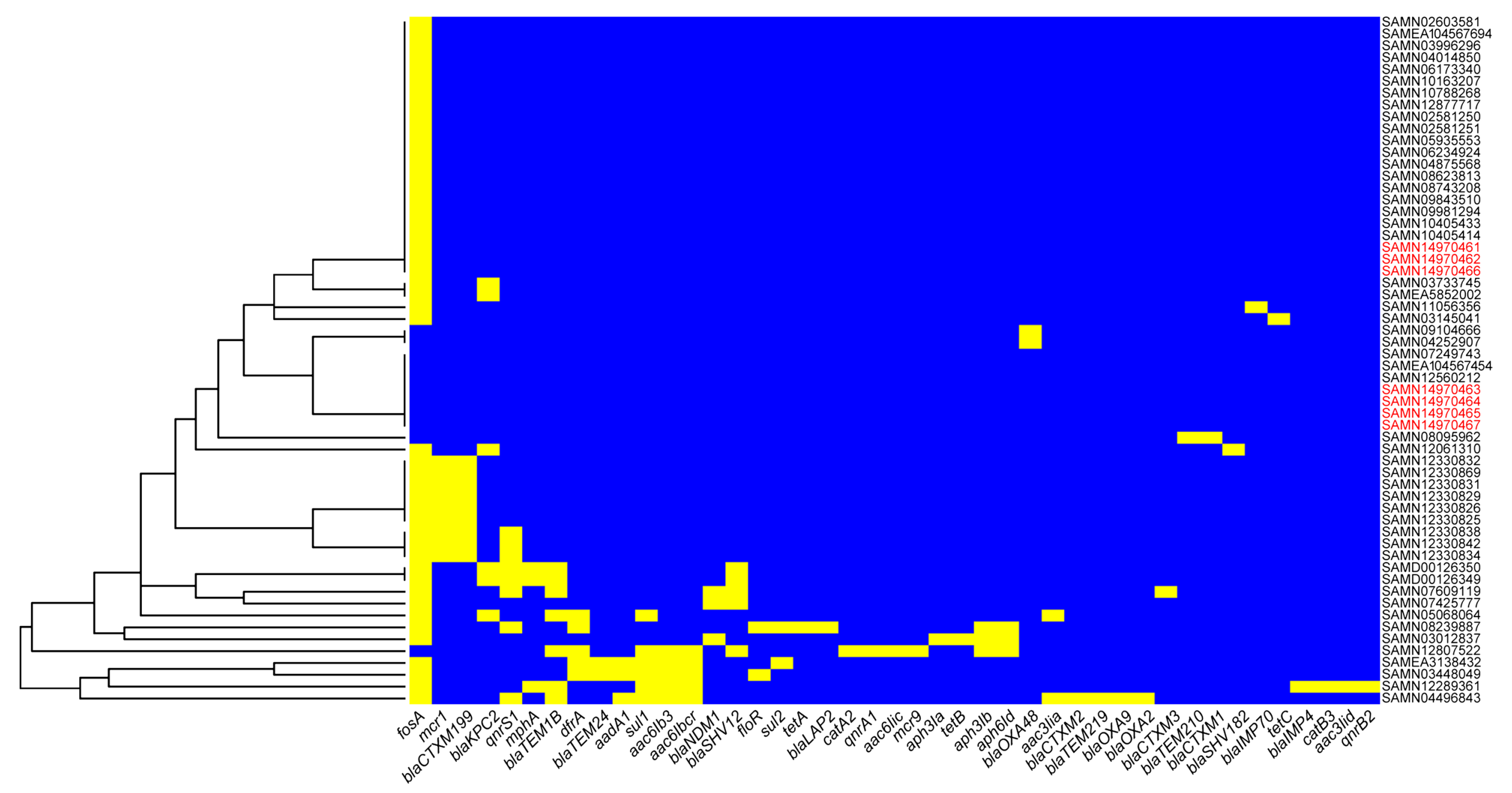

Fig. 2. Antimicrobial resistance genes were identified in the genomes of Klebsiella aerogenes isolates by analyzing the WGS data. The antimicrobial resistance genes (ARGs) are shown on the bottom.

Yellow indicates the presence of the ARGs, and blue indicates the absence of the ARGs. 
bronchoscope suction valves, Lyon, France, 2014. Euro Surveill. 2016 Jul 14;21(28):21.

https://doi.org/10.2807/1560-7917.ES.2016.21.28.30286

Jørgensen SB, Bojer MS, Boll EJ, Martin Y, Helmersen K, Skogstad M, Struve C. Heat-resistant, extended-spectrum $\beta$-lactamase-producing Klebsiella pneumoniae in endoscope-mediated outbreak. J Hosp Infect. 2016 May;93(1):57-62.

https://doi.org/10.1016/j.jhin.2016.01.014

Kirschke DL, Jones TF, Craig AS, Chu PS, Mayernick GG, Patel JA, Schaffner W. Pseudomonas aeruginosa and Serratia marcescens contamination associated with a manufacturing defect in bronchoscopes. N Engl J Med. 2003 Jan 16;348(3):214-220. https://doi.org/10.1056/NEJMoa021791

Liao W, Quan J, Liu L, Zhao D, Jiang Y, Du X, Zhao F, Yu Y, Zhou Z. New insights into the mechanisms of colistin resistance in Klebsiella aerogenes of clinical origin. Int J Antimicrob Agents. 2020 Jun;55(6):105990.

https://doi.org/10.1016/j.ijantimicag.2020.105990

Liu Q, Liu L, Li Y, Chen X, Yan Q, Liu W. Fecal carriage and epidemiology of carbapenem-resistant Enterobacteriaceae among hospitalized patients in a university hospital. Infect Drug Resist 2019 Dec;12:3935-3942. https://doi.org/10.2147/IDR.S233795

Ma DY, Huang HY, Zou H, Wu ML, Lin QX, Liu B, Huang SF. Carbapenem-Resistant Klebsiella aerogenes clinical isolates from a teaching hospital in Southwestern China: detailed molecular epidemiology, resistance determinants, risk factors and clinical outcomes. Infect Drug Resist. 2020 Feb;13:577-585.

https://doi.org/10.2147/IDR.S235975

Malek A, McGlynn K, Taffner S, Fine L, Tesini B, Wang J, Mostafa H, Petry S, Perkins A, Graman P, et al. Next-generation-sequencing-based hospital outbreak investigation yields insight into Klebsiella aerogenes population structure and determinants of carbapenem resistance and pathogenicity. Antimicrob Agents Chemother. 2019 Jun;63(6):63. https://doi.org/10.1128/AAC.02577-18 Miao M, Wen H, Xu P, Niu S, Lv J, Xie X, Mediavilla JR, Tang YW, Kreiswirth BN, Zhang X, et al. Genetic diversity of carbapenem-resistant Enterobacteriaceae (CRE) clinical isolates from a tertiary hospital in Eastern China. Front Microbiol. 2019 Jan 15;9:3341. https://doi.org/10.3389/fmicb.2018.03341

Passarelli-Araujo H, Palmeiro JK, Moharana KC, PedrosaSilva F, Dalla-Costa LM, Venancio TM. Genomic analysis unveils important aspects of population structure, virulence, and antimicrobial resistance in Klebsiella aerogenes. FEBS J. 2019a Oct; 286(19):3797-3810. https://doi.org/10.1111/febs.15005

Passarelli-Araujo H, Palmeiro JK, Moharana KC, Pedrosa-Silva F, Dalla-Costa LM, Venancio TM. Molecular epidemiology of 16S rRNA methyltransferase in Brazil: RmtG in Klebsiella aerogenes ST93 (CC4). An Acad Bras Cienc. 2019b;91 suppl 1:e20180762. https://doi.org/10.1590/0001-376520182018762

Price MN, Dehal PS, Arkin AP. FastTree: computing large minimum evolution trees with profiles instead of a distance matrix. Mol Biol Evol. 2009 Jul 01;26(7):1641-1650.

https://doi.org/10.1093/molbev/msp077

Qin X, Yang Y, Hu F, Zhu D. Hospital clonal dissemination of Enterobacter aerogenes producing carbapenemase KPC-2 in a Chinese teaching hospital. J Med Microbiol. 2014 Feb 01;63(2):222-228. https://doi.org/10.1099/jmm.0.064865-0

Shen X, Liu L, Yu J, Cao X, Zhan Q, Guo Y, Wang L, Yu F. Coexistence of $b l a_{\mathrm{NDM}-1}$ and $r m t C$ on a transferrable plasmid of a novel ST192 Klebsiella aerogenes clinical isolate. Infect Drug Resist. 2019 Dec;12:3883-3891.

https://doi.org/10.2147/IDR.S228130

Srinivasan A, Wolfenden LL, Song X, Mackie K, Hartsell TL, Jones HD, Diette GB, Orens JB, Yung RC, Ross TL, et al. An outbreak of Pseudomonas aeruginosa infections associated with flexible bronchoscopes. N Engl J Med. 2003 Jan 16;348(3):221-227. https://doi.org/10.1056/NEJMoa021808

Tian D, Wang B, Zhang H, Pan F, Wang C, Shi Y, Sun Y. Dissemination of the $b l a_{\mathrm{NDM}-5}$ gene via IncX3-type plasmid among Enterobacteriaceae in children. MSphere. 2020 Feb 26;5(1):5.

https://doi.org/10.1128/mSphere.00699-19

Zamani A. Rigid bronchoscopy induced bacterial translocation: is it a real threat? Eur Respir J. 2004 Feb;23(2):352.1-352, author reply 352-353. https://doi.org/10.1183/09031936.03.00114303a

Zankari E, Hasman H, Cosentino S, Vestergaard M, Rasmussen S, Lund O, Aarestrup FM, Larsen MV. Identification of acquired antimicrobial resistance genes. J Antimicrob Chemother. 2012 Nov 01;67(11):2640-2644. https://doi.org/10.1093/jac/dks261 Zerbino DR, Birney E. Velvet: algorithms for de novo short read assembly using de Bruijn graphs. Genome Res. 2008 Feb 21; 18(5): 821-829. https://doi.org/10.1101/gr.074492.107

Zheng B, Xu H, Lv T, Guo L, Xiao Y, Huang C, Zhang S, Chen Y, Han H, Shen P, et al. Stool Samples of acute diarrhea inpatients as a reservoir of ST11 hypervirulent KPC-2-producing Klebsiella pneumoniae. mSystems. 2020 Jun 30;5(3):5.

https://doi.org/10.1128/mSystems.00498-20

Zheng B, Zhang J, Ji J, Fang Y, Shen P, Ying C, Lv J, Xiao Y, Li L. Emergence of Raoultella ornithinolytica coproducing IMP-4 and KPC-2 carbapenemases in China. Antimicrob Agents Chemother. 2015 Nov;59(11):7086-7089. doi:10.1128/AAC.01363-15

Supplementary materials are available on the journal's website. 